$\begin{array}{ll}\text { Italique } & \text { Italique } \\ \text { Poésie italienne de la Renaissance }\end{array}$

XVIII | 2015

Varia

\title{
La tragedia spettacolo cortigiano della Spagna del XVI secolo
}

Josep Lluís Sirera

Traduttore: Laura Volpe

\section{OpenEdition}

Journals

Edizione digitale

URL: http://journals.openedition.org/italique/411

DOI: 10.4000/italique.411

ISSN: 1663-4438

Editore

Librairie Droz

Edizione cartacea

Data di pubblicazione: 1 ottobre 2015

Paginazione: 137-148

ISBN: 978-2-600-01900-2

ISSN: 1423-3983

Notizia bibliografica digitale

Josep Lluís Sirera, «La tragedia spettacolo cortigiano della Spagna del XVI secolo », Italique [Online], XVIII | 2015, online dal 01 octobre 2017, consultato il 01 mai 2019. URL : http:// journals.openedition.org/italique/411 ; DOI : 10.4000/italique.411

(C) Tous droits réservés 


$$
\text { Josep Lluís Sirera }
$$

LA TRAGEDIA S PETTACOLO C ORTIG I A N D E L L A SPAGNA DEL XVI SECOLO 



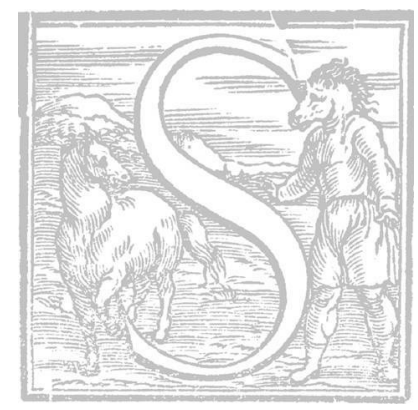

ono passati ormai cinquant'anni da quando il professor Alfredo Hermenegildo pubblicò uno studio notevole sulla tragedia nel teatro spagnolo del XVI secolo (1962). Un decennio più tardi lo rielaborò e lo amplio sostanzialmente grazie, in gran parte, alle opportune considerazioni sull'argomento fatte dal compianto professor Rinaldo Froldi (1973: 94-II5). È superfluo affermare che $i$ contributi di questi due studiosi favorirono una maggiore conoscenza di un periodo della storia del teatro spagnolo sino ad allora poco nota, a parte naturalmente la figura di Juan de la Cueva. Le numerose pubblicazioni dei due professori sono, tra l'altro, servite da sprone per le successive promozioni di altri ricercatori, affinché si sentissero, me compreso, assolutamente attratti dall'affascinante mondo della tragedia.

\section{LA TRAGEDiA NEL TEATRO SPAGNOLO DEL XVI SECOLO, UN GENERE DA RIVISITARE}

Ciò significa forse che possediamo informazioni esaurienti sulle tragedie del teatro spagnolo rinascimentale? Tutt'altro. A differenza di quanto succede, per esempio, nelle tragedie del teatro italiano, le notizie e $i$ documenti su cui abbiamo via via costruito le nostre nozioni si basano, in gran parte (per non dire del tutto), sui testi drammatici, conservati molto spesso sotto forma di pubblicazioni. Possediamo invece pochi dati sulle circostanze della rappresentazione di queste opere. Il fatto poi che vari tragediografi spagnoli svolgessero la loro attività al di fuori della Penisola Iberica non ha certamente aiutato. ${ }^{\mathrm{I}}$ Si può persino dire che soltanto chi si dedicò alla docenza universitaria ci abbia fornito dati più o meno concreti sulla stesura e sulla rappresentazione delle loro opere. ${ }^{2}$ Non aiuta nemmeno il fatto che gli studi sul teatro spagnolo trattassero di preferenza, almeno dall'epoca romantica fino alla genesi del nostro teatro nazionale o, se si preferisce, alle origini della commedia barocca. Molto è ciò che sappiamo al riguardo e molto è ciò che abbiamo via via scoperto negli ultimi cinquant'anni, sia in quanto agli autori e alle opere rappresentative, sia in quanto alle circostanze della loro rappresentazione. ${ }^{3}$ E logico, vista la straordinaria rilevanza del teatro spagnolo nel 
corso del XVI e del XVII secolo; un teatro legato strettamente a quello che possiamo qualificare come pratica scenica professionale o populista, ${ }^{4}$ in modo che il grosso delle ricerche più recenti si ̀̀ centrato sulla delimitazione degli spazi della rappresentazione, sulla conformazione di compagnie professionali, sulle genealogie dei comici, sulle tecniche delle messe in scena, e via dicendo.

Purtroppo, altri aspetti sono stati sviluppati di meno, in parte a causa della difficolta che riveste lo studio delle fonti documentali e della loro scarsità, $e$ in parte a causa dell'esistenza di alcune opere di riferimento che continuano tuttora a fungere da indiscusse pietre miliari. Pensiamo per esempio alle manifestazioni del teatro religioso spagnolo, molto più ricco e variegato di quanto facciano supporre gli autos sacramentales; oppure, per non dilungarmi troppo, alle manifestazioni del teatro cortigiano.

Quest'ultimo caso, a proposito, colpisce alquanto poiché, com'è risaputo, una delle basi su cui si fondò lo sviluppo del teatro castigliano a partire dall'ultimo terzo del XV secolo, fu il teatro rappresentato alle corti dell'epoca, e in special modo in quelle di Re Giovanni II e dei Re Cattolici, nonché alle corti dei grandi nobili come quella di Salamanca dei Duchi di Alba.' La stessa produzione drammatica di Enzina rivela, inoltre, che questo teatro cortigiano non si limitava a spettacoli prevalentemente visivi, in definitiva dei fasti, ma che aveva una stretta relazione con manifestazioni letterarie, soprattutto poetiche: tutti sanno, per esempio, che il drammaturgo di Salamanca raccolse inizialmente le sue opere drammatiche nel suo canzoniere poetico. In effetti, un'accurata rivisitazione dei poemi raccolti nei canzonieri di tale periodo ha rivelato il carattere drammatico di alcuni dei poemi che in essi riscontriamo. ${ }^{6}$

Questo tipo di teatro si mantenne, ovviamente circoscritto all'ambito cortigiano, anche nel corso dei secoli successivi, sebbene abbia suscitato meno interesse di quanto fosse stato auspicabile. ${ }^{7} E$ pensare che le notizie di cui disponiamo ci parlano chiaramente dell'esistenza di importanti centri di questo teatro cortigiano, come quello alla corte del vicereame di Valencia della prima metà del XVI secolo, in cui le figure di spicco erano la regina Germana de Foix e Fernando di Aragona, duca di Calabria. Una corte presso la quale, d'altra parte, il teatro e la letteratura fiorirono in modo realmente singolare. ${ }^{8}$ La mancata considerazione della loro autonomia estetica e politica ha impedito la loro diffusione, ma va detto che di fronte alla potentissima corte reale 
spagnola (stabile dall'ascesa al trono di Filippo II), le corti aristocratiche del XVI secolo non sembravano un granché, ad eccezione di quella valenciana del duca di Calabria, appartenente alla dinastia dei Re di Napoli e sposatosi in prime nozze con Germana de Foix, nipote di Luigi XII di Francia e vedova di Ferdinando il Cattolico. Va detto che, pur essendo il duca di Calabria un viceré per mandato reale, Carlo I lo mantenne in carica finché visse ed ebbe sempre nei suoi confronti una speciale deferenza.

Ciononostante, non si può negare che molte di queste corti nobiliari acquisirono un'importanza e un peso economico, culturale e politico per nulla trascurabile e, in una certa misura, simile a quello di alcuni stati italiani coevi. Pensiamo per esempio a quella del Duca di Lerma e Marchese di Denia, prima ministro di Filippo III, e la cui relazione con il mondo dei fasti è stata studiata da Teresa Ferrer (I99I). Meno conosciute (per non dire per niente conosciute) furono le corti dei viceré valenciani posteriori al Duca di Calabria. La stessa Teresa Ferrer ci ricorda al riguardo, nello studio sopra citato, che nella seconda metà del $X V I$ secolo furono viceré a Valencia e in questo ordine, Alfonso di Aragona duca di Segorbe (I558-1563), Antonio Alfonso Pimentel, conte di Benavente (I566-I575), Vespasiano Gonzaga, principe di Sabbioneta (I575-I578) e Francisco di Moncada, marchese di Aytona (I580-I597), tutti rappresentanti dell'alta nobiltà spagnola e italiana, e che vantavano in alcuni casi strette relazioni con l'attività teatrale di quegli anni. $^{9}$

\section{IL PROBLEMA DELLA RAPPRESENTAZIONE DELLE TRAGEDIE}

Quanto detto finora ha un chiaro significato poiché, come ho fatto notare, una delle grandi carenze rilevabili negli studi sulla tragedia spagnola rinascimentale è la mancanza di informazioni riguardanti le circostanze della rappresentazione della maggioranza delle opere; e pertanto, a prescindere dalle tragedie nate negli ambiti accademici (García Soriano, I945 o Alonso Asenjo, 1995), in tutti gli altri casi non possiamo fare altro che supporre che vennero messe in scena nei teatri pubblici o in spazi privati, senza poter fornire, almeno per il momento, dei dati che possano farci propendere per una o per l'altra possibilità. Possiamo per esempio riscontrare tale lacuna nel caso delle opere scritte da Cristóbal de Virués, uno dei tragediografi fondamentali nella 
Spagna dell'ultimo terzo del XVI secolo. Non mi dilungherò sugli aspetti biografici né sugli aspetti più rimarchevoli della sua pratica scenica poiché disponiamo di una notevole bibliografia al riguardo, ${ }^{\text {'० }}$ ma lo farò sui suoi Prólogos e sui suoi epiloghi che, in quattro delle sue cinque opere, sono a carico del personaggio Tragedia. ${ }^{\text {I }}$ Questi frammenti, nonostante la loro brevità, contengono notizie molto interessanti sulle probabili circostanze della loro rappresentazione e della data della loro stesura. Si sa, per esempio, come nel Prologo de La gran Semíramis Virués rivendichi la novità di essere stato il primo drammaturgo spagnolo a dividere l'opera in tre giornate. ${ }^{\mathrm{I} 2}$ Dato che le sue tragedie sono giunte a noi in una edizione datata I600, ${ }^{13}$ e cioè quando la commedia spagnola si trovava in piena espansione grazie a Lope de Vega, tale attribuzione si poteva sostenere solo sul fatto che Virués avesse scritto quest'opera (e presumibilmente le altre quattro) in date alquanto precedenti; ${ }^{\mathrm{I}}$ la qual cosa giustificherebbe che Lope de Vega affermasse in quello stesso I609 che il Virués fosse stato il primo drammaturgo a scrivere opere in tre atti. ${ }^{15}$

Meno chiare risultano tuttavia le affermazioni di Virués in tali frammenti sui probabili destinatari delle opere. Si tratta anche di versi molto noti tra $i$ quali mi piacerebbe citare $i$ seguenti:

Solo advierto

que no se podrá ver en modo alguno

lo que della prometo, si el silencio

y la atención devida no se guarda.

(Cruel Casandra. Juliá Martínez, I, p. 59 a)

Sólo pide el silencio acostumbrado, aunque pedille en tan illustre parte también, pues es tan cierto, es escusado. (Atila Furioso. Juliá Martínez, I929: 93 a)

Anche se sono senz'altro questi $i$ versi dell'ultima delle opere da egli scritte (La infelice Marcela) quelli che più hanno conquistato $i$ critici e gli storici del teatro.

Salgo con voluntad i firme intento

de procurar el gusto i el regalo

del que con claro entendimiento 
conoce lo que es bueno i lo que es malo;

i luego de través el vano viento

del vulgo, cuyo voto al aire igualo,

me levanta la mar, pensando cierto

que estorba de tomar el salvo puerto.

Esto es assí; mas gran consuelo tengo

pues [h] an de ser en mi favor los sabios,

a quien, pues tales son, nada prevengo

de lo que [h] an de esplicar mis torpes labios;

con los que no lo son, en nada vengo,

ni temo sus satíricos ressabios,

pues aunque en rota barca en su mar ande,

es el favor de los discretos grande.

(Infelice Marcela. Juliá Martínez, I929: I, I I 8 b- I I 9 a)

Va detto che frasi come quei satíricos ressabios di un volgo generico non lasciano tuttavia molti dubbi su qualche probabile rappresentazione (di questa o di un'altra opera del Virués) davanti ad un pubblico ben diverso da quel senado e da quei sabios che per il drammaturgo sarebbero dovuti essere $i$ destinatari ideali delle sue tragedie. ${ }^{16}$ Ma c'è dell'altro, le allusioni alla belligeranza del volgo, che esagerò cercando di far naufragare la nave (e cioè la rappresentazione), ci porta appieno nel terreno del teatro professionale della fine del XVI secolo, dominato da un pubblico che si recava alla rappresentazione mediante il pagamento di un biglietto e senza nessun tipo di coazioni ideologiche o sociali.

Che poi Virués compari questa ricettività negativa (subita e / o temuta) con quella favorevole concessa dai cultori del teatro, potrebbe essere attribuito - a mio avviso- al fatto che il nostro drammaturgo abbia conosciuto tutte e due le platee, o che almeno ebbe l'occasione di essere presente a rappresentazioni conclusesi in un senso o nell'altro. Dato inoltre che Virués risiedette per parecchi anni nella Penisola italiana, anche se pubblicò le sue opere in Spagna, si può ipotizzare che forse le sue tragedie siano state rappresentate durante il suo soggiorno italiano in un ambiente cortigiano o erudito (in qualsiasi caso, minoritario) e che ben altro ricevimento abbiano avuto in Spagna. ${ }^{17}$ In questo caso, non possiamo scartare la possibilità che tali rappresentazioni realizzate presumibilmente davanti ad un pubblico ben predisposto abbiano avuto luogo in Spagna, addirittura nella sua Valencia natia, per quello che commenterò dopo. 
Accettiamo, con le dovute precauzioni, tale possibilità. Dove vennero allestite tali rappresentazioni? Lasciando da parte $i$ corrales, un modello di teatro commerciale regolato da leggi puramente economiche, abbiamo due alternative: innanzitutto $i$ coliseos, che Arróniz ricollega, e secondo me con fondamento, ai teatri delle corti italiane contemporanee (1978). Progettati ad immagine e somiglianza dei teatri cortigiani dell'Italia, $i$ coliseos vennero sicuramente destinati ad ospitare spettacoli per un pubblico scelto, per un senado ilustre, parafrasando il Virués. Ben lontani sono ormai $i$ tempi in cui gli studiosi del teatro spagnolo del XVI secolo contemplavano come modello di spazio di rappresentazione unicamente quello del corral. Lo stesso Arróniz, nel suo lodevole studio or ora citato, dimostrò in modo più che attendibile che la valenciana casa de comèdies de l'Olivera fu ricostruita nel I6I8 in base al modello del Teatro Farnese di Parma (1978: 124-27). In un secondo tempo abbiamo via via scoperto altri locali in tutta la Spagna (soprattutto nei territori della Corona di Aragona) che si avvicinavano al modello italiano, se non addirittura ad esso ispiratisi direttamente. ${ }^{18}$

Nel caso concreto del Virués (e in quello di Rey de Artieda, a cui dedicherò la seconda parte del mio articolo), tuttavia, non possiamo supporre che le eventuali rappresentazioni delle sue tragedie abbiano avuto luogo ne L'Olivera poiché, come abbiamo già visto, le date non combaciano. ${ }^{19}$ E allora, dove? Viene quasi da pensare che in qualche sala dei palazzi della nobiltà valenciana: sale ${ }^{20}$ come quelle che sicuramente accolsero - fasti a parte - le rappresentazioni verso il IS2I, ${ }^{2 \mathrm{I}}$ delle commedie di imitazione celestinesca, Thebayda, Ypólita e Serafina (Canet, 2003); oppure quella che ospitò nel I 34 l'interessantissima Farsa a manera de tragedia (Rodrigo, 1984), in un contesto festivo su cui tornerò immediatamente. Come succedeva nelle corti italiane, insomma, anche nei palazzi della nobiltà valenciana non erano rari non soltanto i fasti spettacolari in sé e per sé, ma anche giochi letterari raffinati, impregnati di spirito rinascimentale e che inclusero - perché noanche rappresentazioni con dei testi drammatici di raffinato estro letterario.

Fu il caso della Farsa a manera appena citata che, nel suo prologo, contiene allusioni a spazi piuttosto concreti della città di Valencia, come afferma Williams nel 1935: «A shepherd open the play with an introit. Statements that he is arriving in the city, that he is a traveler, and that 
it is Sr, John's days, and a reference to the market-place square in Valencia on this day) (I935: I2I). Non voglio assolutamente affermare che quest'opera sia stata rappresentata in tale piazza (uno spazio, a proposito, riservato ai fasti cortigiani come $i$ tornei, nonché alle esecuzioni pubbliche e agli autodafé), ma è più che plausibile che venisse rappresentata in occasione della festa di San Giovanni in alcuni dei luoghi in cui si riunivano $i$ nobili. ${ }^{22}$

Va osservato al riguardo che la nobiltà valenciana (e, per estensione, quella spagnola) della seconda metà del XVI secolo si trovava a mille miglia di distanza da quella dei suoi avi, in quanto a cultura e gusti. Molti dei suoi esponenti coltivavano le lettere con assiduità, anche se non sempre con destrezza. E mantenevano tertulias o, se si preferisce, Academias come succedeva nella vicina Penisola. Limitandoci al territorio valenciano, è sommamente significativo che nel Is92 nascesse nella capitale del Regno di Valencia la Academia de los Nocturnos e che si mantenesse molto attiva fino al $15944^{23}$ Il Canzoniere di questa Accademia, che occupa un posto di assoluta rilevanza nell'ambito delle accademie spagnole, ${ }^{24}$ ci rivela un'intensa attività poetica e retorica, anche se apparentemente non teatrale, nonostante l'elenco degli accademici includa drammaturghi cruciali del teatro spagnolo del periodo: Andrés Rey de Artieda, Francisco Agustín Tárrega o Guillem de Castro, tanto per citarne alcuni. ${ }^{25}$ Erano previsti spettacoli teatrali riconducibili agli accademici? La domanda rimane nell'aria; e allo stesso modo resta in sospeso il tema di eventuali rappresentazioni alle corti del vicereame posteriori a quella del Duca di Calabria e Germana de Foix.

\section{La tragedia Los amantes di Andrés Rey de Artieda}

Nonostante tutto, si possono riscontrare indizi di rappresentazioni di tragedie in ambiti cortigiani. La stessa Farsa a manera de tragedia può essere portato ad esempio e, a mio avviso, lo è anche l'unica opera teatrale che si conserva di Andrés Rey de Artieda, e cioè la tragedia Los Amantes.

Andrés de Artieda (I549-I6I3 ca.) fu militare di professione come il Virués anche se, diversamente da quest'ultimo, risedette piu tempo in Spagna, e soprattutto nella sua Valencia natia. Dottore in Diritto, si dedicò prestissimo alle lettere e, nel I58I, fece stampare una tragedia, 
Los Amantes. Colpisce certamente la sua edizione sciolta, un fatto per nulla abituale all'epoca, ma soprattutto il suo lungo prologo (rivolto Al Ilustre señor don Thomas de Vilanoua) che è un piccolo trattato in terzine sulla tragedia e in cui difende - contrariamente ai precetti classici - una visione molto più innovativa del genere tragico accordato allo stile spagnolo dell'epoca. ${ }^{26}$

Ha altresi interessato alla critica l'argomento dell'opera: è stata la prima versione teatrale di una leggenda ben nota in Spagna, e cioè quella degli amanti di Teruel. ${ }^{27}$ Non ci interessa, tuttavia, il fatto che probabilmente si tratti della prima drammatizzazione di un tema leggendario spagnolo, e nemmeno che le sue origini non risalgano a quella città aragonese, ma che si ispirino ad una storia di ambito locale: quella degli amanti di Bicorp (Merimée, 1985: 300-303), una località valenciana abitata da moriscos di cui era signore precisamente la persona a cui era dedicata l'opera: don Tomás Vilanova. Il nostro interesse nasce piuttosto da una serie di aspetti strutturali che inseriscono l'opera in un contesto di festeggiamenti che, va detto, non siamo in grado né di individuare né di datare, e quindi - a scanso di equivociammettiamo di muoverci in un terreno piuttosto ipotetico, ma per nulla infondato.

A quali aspetti ci riferiamo? In primo luogo, la struttura stessa dell'opera ci apporta alcuni elementi degni di riflessione: ${ }^{28}$ e non tanto per la sua divisione in quattro atti invece dei cinque secondo i canoni del classicismo, o dei tre in cui si dividerà la stragrande maggioranza del teatro spagnolo a partire dagli ultimi anni del XVI secolo, ma per la sua accurata segmentazione degli atti in quadri, come ci fa notare Ojeda Calvo. Ricordiamo che il concetto di quadro comporta la rottura della coerenza argomentale, il cambio nella configurazione dei personaggi, e $i$ salti nel tempo e nello spazio: un qualcosa che contrasta con gli schemi imperanti nella commedia dei secoli d'oro e nelle impostazioni dei tragediografi come il Virués che si propongono di rispettare le tre unità nell'insieme dell'opera o, per lo meno, all'interno dei tre atti in cui le si divide. ${ }^{29}$ Più che di un'apparente anomalia, bisognerebbe qui parlare dell'utilizzo di uno dei procedimenti abituali della pratica scenica cortigiana il cui fine ultimo sarebbe quello di intercalare durante la rappresentazione degli intermezzi piuttosto variati poiché, va tenuto presente, l'opera teatrale farebbe parte di un insieme festivo molto più esteso e complesso. 
Naturalmente l'abilità dell'autore consisteva nel programmare la fine di ogni atto in modo da poterla riallacciare agli intermezzi festivi. Cosi dunque, il primo atto finisce in un modo alquanto rivelatore: un banditore pubblica un avviso per annunciare un torneo in cui vengono sfidati i cavalieri presenti agli sponsali di Isabel de Segura e tra i quali si trova il suo afflitto e deluso spasimante, Diego Marcilla. Si tratta di una pratica tipica del teatro cortigiano a cui era solito ricorrere un altro drammaturgo valenciano dell'epoca, il canonico Francisco Agustín de Tárrega, autore di opere come Las suertes trocadas y torneo venturoso, dal titolo piuttosto esplicito, non c'è che dire. ${ }^{30}$

Ma c'è dell'altro: il secondo atto si chiude con una cena vivacizzata da cantori: «Entrémonos a cenar / y vosotros cantad algo» (Juliá Martinez, I929: I, I2b) che, come alla fine del primo atto, ha ben poco a che vedere con il tono che si addice ad una tragedia. L'autore poi, nel suo desiderio di legare al massimo la trama con i motivi della rappresentazione (cioè con i festeggiamenti che vi si intercalano o, se si preferisce, con quelli che scandiscono la tragedia), fa in modo che i protagonisti, nonostante stiano vivendo una situazione di assoluto dolore, ${ }^{31}$ si comportino secondo $i$ clichés cortigiani, nascondendo dietro di essi lo sconforto e il turbamento che vivono.

Il tono tragico, è vero, si imporrà da quel momento in poi: in effetti, il terzo atto si chiude con la morte di Marcilla, e il quarto con un'esaltazione dei due amanti dopo la morte di Isabel de Segura. Purtuttavia, possiamo trovare tracce del concetto cortigiano della rappresentazione teatrale in questa seconda parte dell'opera. Stupisce per esempio che la morte di Diego Marcilla avvenga fuori dalla scena e che gli spettatori vi assistano per interposta persona. La morte del giovane infatti ci viene narrata da una delle damigelle di Isabella che spia quello che lei crede sia il primo incontro appassionato tra i novelli sposi e che interpreta erroneamente $i$ lamenti di Diego come effusioni d'amore degli sposi, anche se a lei, invero, sembrano pervase di tristezza. La critica ba sottolineato tale aspetto come un elemento che smorza la tragicità che la rappresentazione sta acquisendo, ma non ha prestato attenzione invece al fatto che si tratta di una scena che gioca abilmente con il dentro / fuori caratteristico del teatro cortigiano che, in mancanza di uno spazio di rappresentazione standardizzato, sfrutta le disponibilità dello spazio scenico della sala o del cortile in cui si presenta l'opera per giocare con ciò che si vede, ciò che si suggerisce o, semplicemente, ciò che si narra, come 
- per esempio - ha sottolineato Canet riguardo alle commedie Thebayda e Seraphina (1984: 291-93). Detto altrimenti: abbiamo un adeguamento, uno dei tanti, della tragedia Los Amantes alle circostanze cortigiane della rappresentazione.

In definitiva, dalla succinta analisi della struttura della tragedia di Rey de Artieda, e dalle informazioni fornite da Cristóbal de Virués sul suo pubblico (reale o vagheggiato), si deduce che, indipendentemente dal fatto che ci riuscissero o meno, $i$ due tragediografi concepirono le loro opere per destinarle ad un pubblico colto e di un ceto sociale alto: è per questo che si spiega la solidità drammatica che presentano. E questo perché nell' ambiente cortigiano (a parte i circoli universitari, che non sono stati oggetto di una meritata attenzione in questo articolo) la rappresentazione delle opere di questo tipo non era un qualcosa di strano, bensi tutto il contrario. Ci fu, in definitiva, un pubblico cortigiano e colto per queste opere nella Valencia dell'ultimo terzo del XVI secolo, degli spazi in cui era possibile presentare tali spettacoli ed esistevano le circostanze concrete che li giustificavano: le feste che si celebravano presso le corti nobiliari e - probabilmente - vicereali del Regno di Valencia durante tale periodo.

Josep Lluís Sirera (traduzione dallo spagnolo di Laura Volpe) 
I. In effetti, drammaturghi come Andrés Rey de Artieda, Cristóbal de Virués o Gabriel Lobo Lasso de la Vega si dedicarono alla carriera delle armi e trascorsero parecchi anni fuori dalla Penisola. A ciò si deve che Arróniz (1978: 175-76) parli della generazione dei capitanes de Lepanto per riferirsi a questi autori.

2. Come nel caso di Jerónimo Bermúdez. Vedi per esempio Hermenegildo (1973: 163-92) e Triwedi (1975).

3. L'importanza e la consistenza di questi studi in questi ultimi decenni sono stati tali che le antologie come quella coordinata dal professor Jean Canavaggio (I995) sono state superate, in alcune aree, dalle ricerche più recenti.

4. Riguardo al concetto di práctica escénica e, più concretamente, riguardo a quella populista, vedi Quirante-Rodríguez-Sirera (1999: I 25-52).

5. Abbiamo un'abbondante bibliografia sul teatro cortigiano castigliano della fine del XV e inizio del XVI secolo, imperniato sulla singolare figura di Juan del Enzina (ma non solo su di essa). Senza avere la pretesa di essere esauriente, mi permetto di ricordare i classici lavori di Surtz (I979), Rosalie Gimeno (1976 e I 977) o Miguel Ángel Pérez Priego (i 99 I e 1997), nonché di Gutiérrez Ceballos, ed (1998).

6. Vedi per esempio: Sirera (1992) e Rodado (1995).

7. Con pregevoli e rilevanti eccezioni, come lo studio imprescindibile di Teresa Ferrer (I99I). Purtroppo, questa studiosa si interessa prevalentemente della pratica scenica cortigiana tra il XVI e il XVII secolo e sua relazione con lo sviluppo e consolidamento della stessa commedia.

8. Sirera (1984, I986c e 2000); Oleza (1984).

9. Teresa Ferrer (I991: 55-57) espone, in effetti, lo stretto legame del conte di Benavente con il nascente teatro professionale castigliano (divenne famosa la lite che ebbe con la moglie di Lope de Rueda), e l'interesse che dimostrò per il teatro il principe di Sabbioneta, per il quale Scamozzi costruì un teatro nella città di Sabbioneta.

ı. Oltre agli studi di Hermenegildo e di Froldi, possiamo citare - tra gli altri quelli già classici di Merimée (I985), Sargent (1930), Weiger (1978 e I985) o Sirera (1981, I986b), ai quali vanno aggiunti quelli più recenti di Hermenegildo (I998, 2003) o di Romera Pintor-Sirera (2008).

I I. Sono queste quattro opere: La gran Semiramis, La cruel Casandra, Atila furioso e La infelice Marcela. Nella quinta delle sue tragedie, Elisa Dido, non esiste questo personaggio di chiusura perché, essendo stata scritta l'opera secondo le norme classiche, è il Coro che conclude l'opera.

I 2. «Ni es menor novedad que la que dixe / de ser primera en ser de tres jornadas» (in Juliá Martínez, I 929: I, 26). Citerò sempre questa edizione. 
I 3. Le tragedie si trovano all'inizio delle Obras trágicas y líricas del Capitán Cristoual de Virués. Madrid: a casa di Alonso Martín, i6o9.

I4. Vi è consenso nel datare la loro stesura tra la fine degli anni Settanta e la prima metà degli anni Ottanta del XVI secolo. Vedi per esempio: Froldi (I973: i I I) e Sirera (I986b: 7 I-73).

I 5 . «El capitán Virués, insigne ingenio, / puso en tres actos la comedia, que antes / andaba en cuatro, como pies de niño» (LOPE DE VEGA, Arte nuevo de hacer comedias, vv. 2I 5-I 7). Citato da Rodríguez (201 I: 3I 8-3 I9).

\section{I6. Romera Pintor-Sirera (2008: 69-72)}

17. Senza escludere le due possibilità in Spagna, evidentemente. Più suggestiva sarebbe l'ipotesi secondo cui, essendo Virués consapevole del carattere minoritario delle sue opere precedenti a La infelice Marcela, cercasse di scriverla pensando al pubblico predominante dei corrales. La reazione negativa di quest'ultimo nei confronti dell'opera giustificherebbe il carattere molto più belligerante di questo Prologo e, se accettiamo (come sembrano indicare gli studi cronologici citati nella nota I 4) che questa fu la sua ultima opera, questo forse spiegherebbe anche perché Virués non abbia continuato la sua carriera come drammaturgo.

I 8. Nel I995, io stesso parlavo dell'esistenza di modelli assimilabili ai coliseos, sia per motivi climatologici (un teatro coperto come il coliseo risultava più utile nella Spagna piovosa, per esempio), sia per motivi sociali (uno spazio destinato ad ospitare preferibilmente gli esponenti più in vista della società), sia per un desiderio di grandiosità, molto più facile da soddisfare in locali di questo tipo.

I9. È vero che potremmo ipotizzare una loro rappresentazione nell'originario teatro de La Olivera, dato che cominciò a funzionare nel i 584 , ma non possiamo nemmeno dimenticare che questo primo teatro era più simile ai corrales che ai coliseos propriamente detti (Sirera, I 986c). L'argomento, tuttavia, continua a rimanere nel vago.

20. Con il termine sala, mi riferisco in modo generico ai vari spazi dei palazzi mediterranei in cui era possibile allestire delle rappresentazioni teatrali: gli estrados, fulcro della vita sociale e in cui imperava l'etichetta, ma anche i numerosi saloni in cui si svolgeva ogni tipo di attività, soprattutto quelle ludiche. Senza dimenticare, naturalmente, il patio central, il cortile con i suoi vari livelli, finestre, scale, ecc. Per il caso italiano, equiparabile - credo - a quello valenciano, vedi Molinari (1964).

21. Queste tre opere vennero in effetti pubblicate nel i 5 2 I a Valencia (dal tipografo Jorge Costilla). L'edizione fu dedicata all'allora Duca di Gandia, Joan de Borja i Enríquez, uno dei grandi nobili del Regno di Valencia e imparentato per via materna con la famiglia reale spagnola. Un altro caso di mecenatismo nobiliare del teatro che renderebbe plausibile, a mio avviso, la rappresentazione - o lettura drammatizzata, visto che la Comedia Thebayda è molto lunga - nel palazzo ducale di Gandia, oppure nel palazzo che questa famiglia possedeva nella città di Valencia. 
22. Non dimentichiamo che nel i 534, data dell'edizione di quest'opera, la corte del Duca di Calabria e della Regina Germana de Foix si trovava al massimo del suo splendore, come è stato dimostrato dagli studi di Sirera (i $986 \mathrm{c}$ e 2000).

23. In questa Accademia, in effetti, si diedero appuntamento membri della nobiltà urbana, ciutadans honrats, universitari, funzionari, ecc. Vedi al riguardo: Canet (1 993). La formazione culturale di questi, come dimostra il sopra citato Canzoniere, ci dimostra una cultura vasta e addirittura piuttosto moderna (Sirera, I 993).

24. Il Canzoniere è stato parzialmente editato da Canet-Rodríguez-Sirera (19882000).

25. Non fece parte dell'Accademia, tuttavia, Cristóbal de Virués perché residette per molti anni in Italia, soprattutto nel Ducato di Milano. Questo fatto, tuttavia, non esclude la possibilità che le sue opere circolassero nell'Istituzione poiché di questa Accademia fece parte suo fratello, il medico Jerónimo de Virués.

26. Arcinoti sono, per esempio, i suoi versi: «Digo que España está en su edad robusta. / Y como en lengua, y armas valga, y pueda, / Me parece gustar de lo que gusta» (Juliá Martínez, I929: XXVI). Sulla teoria teatrale di Rey de Artieda: vedi Sirera (i986b: 73-79).

27. Su questa leggenda, molto rappresentata nel teatro spagnolo del XVI secolo, continua ad essere imprescindibile: Cotarelo y Mori, 1907. Anche in Merimée (I985:290-303).

28. Lo studio migliore effettuato finora sulla struttura dell'opera in Ojeda Calvo, 2009. Ad egli ci rifacciamo per completare i commenti che faccio sull'opera.

29. Come indica Virués nel prologo de la Gran Semíramis: «Y solamente, porque importa, advierto / que esta tragedia, con estilo nuevo / que ella introduze, viene en tres jornadas / que suceden en tiempos diferentes [...] / Formando en cada cual una tragedia / con que podrá toda la de [h]oi tenerse / por tres tragedias no sin arte escritas» (Juliá Martínez, i 929: I, $25 b-26 a$ ).

30. Un'altra delle sue opere, La enemiga favorable, ha bisogno di un torneo in scena per la sua conclusione, mentre nell'edizione della sua opera migliore, El Prado de Valencia, all'inizio della seconda giornata, Tárrega inserisce la lunga narrazione di un torneo festivo - come quello che troviamo ne Los Amantes - e che ebbe davvero luogo (Canet e Sirera, i986: I 28-3 I).

3I. Per chi non conoscesse la trama dell'opera, diciamo che l'amore dei due amanti, Isabel e Diego, viene ostacolato dal padre della fanciulla il quale esige dal giovane una ricchezza che egli non possiede. Diego, per rendersi meritevole agli occhi della sua amata, va a lottare contro i musulmani con la speranza di conquistarsi un bottino sufficiente agli occhi del futuro suocero. I giovani fanno un patto: Isabel aspetterà Diego per sette anni. Sebbene il giovane riesca ad accumulare l'agognata fortuna, una serie di sfortunati incidenti causano il ritardo del suo rientro, e precisamente dopo la scadenza del periodo fissato da Isabel. Al rientro, 
quindi, scopre che la ragazza si è appena sposata. Diego, disperato, entra nella camera nuziale e, approfittando di un momento di distrazione del marito, chiede un bacio a Isabel, la quale, però, glielo nega. Diego muore trafitto dal dolore. Il giorno dopo, mentre stanno per inumarlo, Isabel si avvicina al feretro, gli dà il bacio che ore prima gli aveva negato ed ella stessa muore di dolore. Sconvolti da questa dimostrazione di reciproco amore, i familiari di entrambi i giovani e le autorità di Teruel decidono di costruire un mausoleo affinché i due amanti riposino per sempre uno accanto all'altro.

\section{BIBLIOGRAFIA}

Alonso Asenjo, Julio (1995): La tragedia de San Hermenegildo y otras obras de teatro español de colegio. Valencia: Universitat de València.

ArrónIz, Othón (1969): La influencia italiana en el nacimiento de la comedia española. Madrid: Gredos.

Arróniz, Othón (1978): Teatros y escenarios del Siglo de Oro. Madrid: Gredos.

Canavaggio, Jean (I 995): Editore de La comedia. Seminario hispano-francés organizado por la Casa Velázquez: Madrid: Casa Velázquez.

CAnet, José Luis (1984): "La comedia Thebayda y la Seraphina”, in M. V. Diago Moncholí, editore di Teatro y prácticas escénicas. I: el Quinientos valenciano. Valencia: Institució Alfons el Magnànim, pp. 283-300.

CANET, José Luis (1993): "Estructura del saber y estructura del poder: organización y funciones de la Academia de los Nocturnos en Valencia", in E. Rodríguez editrice di De las Academias a la Enciclopedia. Valencia: Institució Alfons el Magnànim, pp. 95- I 24.

CAnet, José Luis (2003): Edizione e introduzione della Comedia Thebayda. Salamanca: Ediciones de la Universidad de Salamanca.

Canet, José Luis e Josep Lluís Sirera (1986): "Francisco Agustín Tárrega”, in J. L. Canet, editore di Teatro y prácticas escénicas. II: la Comedia. Londra: Tamesis Books Limited, pp. го5-I 3 I.

Canet, José Luis, Evangelina Rodríguez e Josep Lluís Sirera (i988-2000): Actas de la Academia de los Nocturnos. Valencia: Institució Alfons el Magnànim, cinque volumi pubblicati.

Cotarelo y Mori, Emilio (1 907): Sobre elorigen y desarrollo de la leyenda de "Los amantes de Teruel'. Madrid: Tipografía della Revista de Archivos, Bibliotecas y Museos.

Ferrer VAlls, Teresa (I 99I): La práctica escénica cortesana: de la época del Emperador a la de Felipe III. Londra: Tamesis Books Limited.

Ferrer Valls, Teresa (1997): Introduzione ed edizione di Teatro clásico en Valencia, I. Madrid: Fundación José Antonio de Castro.

Froldi, Rinaldo $\left(1973^{2}\right)$ : Lope de Vega y la formación de la comedia. Salamanca: Anaya.

García Soriano, Justo (I 945): El teatro universitario y bumanístico en España. Toledo: Talleres de Rafael Gómez-Menor.

Gimeno, Rosalíe (1975): Editrice di Juan del Encina, Obra dramática (I). Madrid: Istmo. 
Gimeno, Rosalíe (1977): Editrice di Juan del Encina, Segunda producción dramática. Madrid: Alhambra.

Gutiérrez Ceballos, Javier (I 999): Editore di Humanismo y literatura en tiempos de Juan del Encina. Salamanca: Ediciones de la Universidad de Salamanca.

Hermenegildo, Alfredo (1962): Los trágicos españoles del siglo XV. Madrid: Fundación Universitaria Española.

Hermenegildo, Alfredo (1 973): La tragedia en el Renacimiento español. Barcellona: Editorial Planeta.

Hermenegildo, Alfredo (1998): Editore di Teatro español del siglo XVI. Madrid: Biblioteca Nueva.

Hermenegildo, Alfredo (2003): Editore de La gran Semíramis e Elisa Dido di Cristóbal de Virués. Madrid: Cátedra.

Juliá Martínez, Eduardo (1929): Studio introduttivo ed edizione di Poetas dramáticos valencianos. Madrid: Real Academia Española, due volumi.

Merimée, Henri (1985): El arte dramático en Valencia. Valencia: Institució Alfons el Magnànim. Prima Edizione (L'art dramatique à Valencia. Depuis les origines jusqu'au commencement du XVII siècle), I9I3.

Molinari, Cesare (1964): "Les rapports entre la scène et les spectateurs dans le théâtre italien du $\mathrm{xvI}^{\mathrm{e}}$ siècle", in J. Jacquot, editore de Le lieu théâtral à la Renaissance. Parigi: Centre National de la Recherche Scientifique, pp. 6i-72.

Ojeda Calvo, María del Valle (2009): "Métrica y estructura dramática en Los Amantes de Andrés Rey de Artieda", in J. Álvarez Barrientos et alteri, coordinatori di En buena compañia. Estudios en honor de Luciano García Lorenzo. Madrid: Consejo Superior de Investigaciones Científicas, pp. 475-487.

OlezA, Juan (1984): "La tradición pastoril y la práctica cortesana, II: coloquios y señores”, in M. V. Diago Moncholí, editore di Teatro y prácticas escénicas. I: el Quinientos valenciano. Valencia: Institució Alfons el Magnànim, pp. 243-258.

Pérez Priego, Miguel Ángel (i 99i): Editore di Juan del Enzina, Teatro completo. Madrid: Cátedra.

Pérez Priego, Miguel Ángel (i 997): Teatro medieval: Castilla. Barcellona: Crítica.

Pérez Priego, Miguel Ángel (2004): El teatro en el Renacimiento. Madrid: Ediciones del Laberinto.

Quirante, Luis, Evangelina Rodríguez e Josep Lluís Sirera (i999): Pràctiques escèniques de l'edat mitjana als segles d'or. Valencia: Universitat de València.

Rodado, Ana (1995): "Poesía cortesana y teatro: posibilidades dramáticas de algunos textos cancioneriles del siglo xv”, in F. B. Pedraza Jiménez e R. González Cañal, editori de Los albores del teatro español. Almagro: Universidad de Castilla La Mancha, pp. 25-44.

Rodrigo, Ricardo (I 984): "Notas en torno a la Farsa a manera de tragedia". In M. V. Diago Moncholí, editore di Teatro y prácticas escénicas. I: el Quinientos valenciano. Valencia: Institució Alfons el Magnànim, pp. 21 9-24I .

Rodríguez, Evangelina (20 I I): Studio ed edizione di Lope de Vega, Arte Nuevo de hacer comedias. Madrid: Castalia.

Romera Pintor, Irene e Josep Lluís Sirera (2008): “Disinganno e moralizzazione 
ne La infelice Marcela di Virués. Sulle fonti giraldiane della sua opera teatrale", in P. Cherchi, M. Rinaldi e M. Tempera, editori di Giovan Battista Giraldi Cinzio gentiluomo ferrarese. Firenze: Leo S. Olschki editore, pp. 53-76.

Sargent, Cecilia V. (1930): A study of the Dramatic Works of Cristóbal de Virués. New York: Instituto de las Españas en los Estados Unidos.

SirerA, Josep Lluís (1986a): "La infraestructura teatral valenciana", in J. L. Canet, editore di Teatro y prácticas escénicas. II: la Comedia. Londra: Tamesis Books Limited, pp. 26-39.

Sirera, Josep Lluís (1986b): "Rey de Artieda y Virués: la tragedia valenciana del Quinientos", in J. L. Canet, editore di Teatro y prácticas escénicas. II: la Comedia, pp. 69-10I.

Sirera, Josep Lluís (1986c): "Espectáculo y teatralidad en la Valencia del Renacimiento". Edad de Oro, V, pp. 247-270.

SiReRA, Josep Lluís (I992): "Diálogos de cancionero y teatralidad", in R. Beltrán, J. L. Canet e J. Ll. Sirera, editori di Historias y ficciones. Valencia: Universitat de València, pp. 35 I-362.

Sirera, Josep Lluís (i 993): "El universo cultural de la Academia de los Nocturnos", in E. Rodríguez editrice di De las Academias a la Enciclopedia. Valencia: Institució Alfons el Magnànim, pp. I 2 5-170.

Sirera, Josep Lluís (1995): "Espectáculo y representación. Los actores. El público. Estado de la cuestión”, in Canavaggio (I995), pp. I I 5-I 30.

Sirera, Josep Lluís (2000): "Jouer à l'amour: fête et littérature dans la Valence du $\mathrm{xvI}^{\mathrm{e}}$ siècle", in D. Jiménez Plaza e J.-Ch. Abramovici, Éros volubile: les métamorphoses de l'amour du Moyen Âge aux Lumières. Parigi: Desjonquères, pp. 70-80.

SurTz, Ronald E. (I97I): The Birth of a Theater. Madrid: Castalia.

Triwedi, Mitchel D. (1975): Primeras tragedias españolas. Madrid: University of North Carolina.

Weiger, John G. (1978): Hacia la comedia: de los valencianos a Lope. Madrid: Cupsa Editorial.

Weiger, John G. (1985): Editore de La infelice Marcela de Cristóbal de Virués. Valencia: Albatros.

Williams, Ronald Boal (1935): The Stanging of Plays in the Spanish Peninsula prior to I555. Iowa City: The University. 